\title{
Structural and Morphological Investigations of $\beta$-Cyclodextrin-Coated Silver Nanoparticles
}

\author{
Patricia Fernanda Andrade, Andreia Fonseca de Faria, Douglas Soares da Silva, \\ Juliano Alves Bonacin, Maria do Carmo Gonçalves
}

\section{Department of Chemical-UNICAMP, Campinas/SP, Brazil}

Metallic nanoparticles are solid particles that range from 1 to $100 \mathrm{~nm}$ in size and which can be found in non-crystalline, nanocrystalline or nanocluster forms [1]. They are usually obtained from colloidal dispersions and, according to the method used during the synthesis, may have a variety of morphologies, such as nanocubes, nanoprisms, nanospheres, triangular nanoplates and wires. Among the various metal nanoparticles, silver nanoparticles (AgNP) have been widely investigated because they exhibit unusual optical, electronic and antimicrobial properties, depending on their size and shape, thus opening many possibilities with respect to technological applications [2]. For example, materials containing AgNP can be used to control the bacterial growth in biomedical devices, dental resin composites, and textile materials [3]. Many studies have recently demonstrated that some molecules presenting hydroxyl groups, such as poly(vinyl alcohol), poly(ethylene glycol), oligosaccharides and polysaccharides, can act as protecting agents to stabilize noble metal particles [4]. $\beta$-cyclodextrin $(\beta-C D)$, with an $0.78 \mathrm{~nm}$ internal cavity diameter, is the most used molecule, however it is too small to entrap AgNP and therefore simply binds, via chemisorptions, to the nanoparticles by means of the rim hydroxyl groups [5]. This paper describes the synthesis of silver nanoparticles using an aqueous silver nitrate solution in the presence of glucose as a reducing agent, sodium hydroxide as a reaction catalyst and $\beta$-CD as a stabilizer. The morphology and especially the stabilizing layer around the silver nanoparticles were studied. The nanoparticles were pseudo-spherical with an average 20-40 nm diameter as observed in transmission electron microscopy images. Complementary analytical electron microscopy based on electron spectroscopy imaging in the transmission microscope was applied to investigate the $\beta$-CD layer surrounding the nanoparticles. Mapping images showed the presence of carbon and oxygen around the nanoparticles, which were attributed to a layer of $\beta-C D$, with approximately $12 \mathrm{~nm}$ thickness. Results of antibacterial tests showed that these silver nanoparticles had promising antibacterial activity against several types of bacterial strains, such as Eschirechia coli, Staphylococcus aureus and Pseudomonas aeruginosa. Curves overtime showed a $20 \mu \mathrm{g} / \mathrm{ml}$ minimum inhibitory concentration for $2 \mathrm{~h}$ exposure to E. coli. This result is promising and points to the possibility of using these nanoparticles as antibacterial additives for different applications, such as antimicrobial food packaging, ultrafiltration membranes and biomedical devices.

\section{References:}

[1] M. Maskos, RH Stauber (2011) Characterization of nanoparticles of biological environments. In: Ducheyne P (ed) Comprehensive Biomaterials, 1st edn. Elsevier, Amsterdam, pp 329

[2] M. Rycenga, C.M. Cobley, J. Zeng, W. Li, C.H. Moran, Q. Zhang, D. Qin, Y. Xia, Chem Rev 111 (2011), p. 3669.

[3] V.D. Lago, L.F. Oliveira, K.A. Gonçalves, J. Kobarg, M.B. Cardoso, J Mater Chem 21 (2011), p. 12267.

[4] P. Vasileva, B. Donkova, I. Karadjova, C. Dushkin, Colloids Surf A 382 (2011), p. 203.

[5] M. Singh, R. Sharma, U.C. Banerjee, Biotechnol Adv 20 (2002), p. 341. 

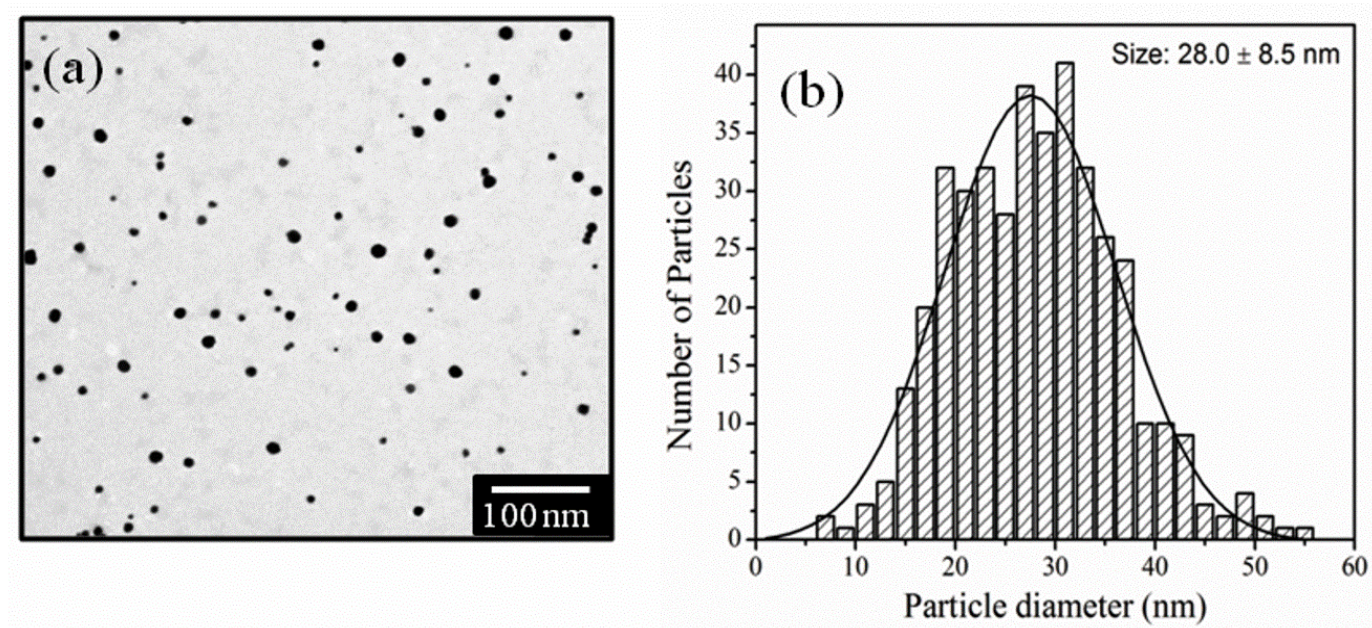

Figure 1. TEM micrograph and the corresponding size distribution histogram of AgNP- $\beta-C D$. The total number of particles was 405

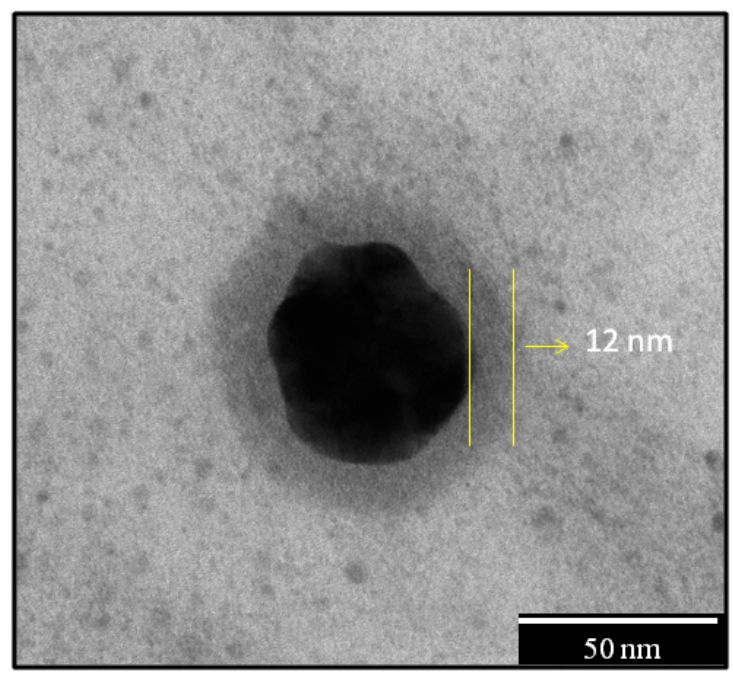

Figure 2. ESI-TEM image of the AgNP- $\beta-C D$ nanoparticle 